Influence of Sedimentation on Crystallization of Charged Colloidal Particles

This article has been downloaded from IOPscience. Please scroll down to see the full text article.

2012 Chin. J. Chem. Phys. 25318

(http://iopscience.iop.org/1674-0068/25/3/11)

View the table of contents for this issue, or go to the journal homepage for more

Download details:

IP Address: 159.226.231.80

The article was downloaded on 11/12/2012 at 23:58

Please note that terms and conditions apply. 


\title{
ARTICLE \\ Influence of Sedimentation on Crystallization of Charged Colloidal Particles
}

\author{
Xuan Du ${ }^{a, b}$, Sheng-hua Xu $u^{a, b}$, Zhi-wei Sun ${ }^{a, b *}$, Lei Liu ${ }^{a, b}$ \\ a. Key Laboratory of Microgravity, Institute of Mechanics, Chinese Academy of Sciences, Beijing 100190, \\ China \\ b. National Microgravity Laboratory, Institute of Mechanics, Chinese Academy of Sciences, Beijing \\ 100190, China
}

(Dated: Received on February 8, 2012; Accepted on April 5, 2012)

The method of density matching between the solid and liquid phases is often adopted to effectively eliminate the effect of sedimentation of suspensions on dynamic behavior of a colloidal system. Experiments on crystallization of charged colloidal microspheres with diameter of $98 \mathrm{~nm}$ dispersed in density-matched and -unmatched media (mixtures of $\mathrm{H}_{2} \mathrm{O}$ and $\mathrm{D}_{2} \mathrm{O}$ in proper proportion) are compared to examine the influence of sedimentation. Reflection spectra of colloidal suspensions were used to monitor the crystallization process. Results showed that the crystal size of the density-unmatched (namely, in the presence of sedimentation) sample grew faster than that of the density-matched (in the absence of sedimentation) case at the initial stage of the crystallization, and then the latter overtook and outstripped the former. To explain these observations, we assume that in the settling of crystals sedimentation facilitates result in more particles getting into the crystal structures. However, as the crystals increase to varying sizes, the settling velocities become large and hydrodynamic friction strips off some particles from the delicate crystal structures. Overall, the sedimentation appears to accelerate the crystal size growth initially and then retard the growth. In addition, the crystal structures formed under microgravity were more closely packed than that in normal gravity.

Key words: Colloidal crystal, Density matching, Sedimentation, Reflection spectrum

\section{INTRODUCTION}

Colloidal crystal is an ordered array of colloidal particles with micron or submicron size. It is analogous to a standard crystal formed by atoms or molecules but the repeating subunits are several orders larger. Therefore colloidal crystals have lately been of great interest to scientists and engineers because they can serve as a model system for studying the behavior of atom crystals on a much larger scale $[1-15]$. Besides, one also expects to better understand how colloid structures grow and behave with the aim of learning how to control colloidal crystal growth to improve the development of novel materials with extraordinary properties [4, 12-15], such as photonic crystal, pressure sensitive sensor, and so on. However, the elastic moduli of colloidal crystals are extremely low and thus even very weak external fields may distort the structure of colloidal crystals. Therefore, in ground-based experiments, sedimentation and convection introduced by gravity may hinder us from being able to gain physical insight into the detailed dynamics characterizing colloidal crystallization. In the

\footnotetext{
* Author to whom correspondence should be addressed. E-mail: sunzw@imech.ac.cn, Tel.: +86-10-82544093
}

early years, Crandall and Williams studied the elastic deformation of colloidal crystal caused by sedimentation and determined the relevant Young's modulus [16]. And then the influences of sedimentation on the crystallization of colloidal particles have been reported in many publications $[17-36]$, including the effects of sedimentation, convection and static pressure derived from gravity on the crystal nucleation and growth, critical phenomena, distortion and collapse of structures, the spatial distribution of aerosols, etc. With the development of space technology, scientists and engineers have been able to further deepen their understanding of some interesting colloid behavior that might appear only under zero gravity conditions. This situation impels us to conduct experiments on colloidal crystallization under a microgravity environment to eliminate the effect of the sedimentation and convection. Among these efforts, microgravity experiments of hard sphere colloidal crystals were performed on the Space Shuttle Columbia. Experiments of colloidal crystal nucleation and growth were also conducted at the International Space Station [19-21]. Under short-term conditions of low gravity, meanwhile, the effects of gravity on the charged colloidal crystal growth were also examined through a series of experiments using parabolic flight or sounding rocket $[22-25]$.

Concerning microgravity experiments, a spacecraft 
in orbit can provide a long-term and relatively stable microgravity environment, but this approach is very costly. On the other hand, parabolic flight and sounding rocket flight provide an economical solution to shortterm low gravity environments but the stability of soobtained low-gravity level is rather limited.

An inexpensive approach for minimizing the effect of sedimentation of suspensions on the dynamic behavior of a colloidal system is the density matching method. In this method, the solvent density is adjusted to match the density of particles so as to make the buoyancy cancel the gravitational force on particles. This method was adopted to effectively eliminate the effect of sedimentation to study the dynamic behavior of colloidal systems $[37-40]$. Besides, when the temperature gradient on the experimental sample is carefully restricted to a small range, the convection in the sample can also be effectively suppressed. In this case, an equivalent microgravity condition can be achieved and its microgravity level is quite stable for a long duration. The advantage of a stable low gravity makes it possible for us to study the time evolution of the mean crystal size for long-duration crystallization with reasonable accuracy.

Okubo et al. conducted a series of experiments for the colloidal crystallization of silica spheres in a shortterm (about $20 \mathrm{~s}$ ) low gravity environment created by parabolic flight and found that rates of nucleation and crystal growth decrease in microgravity [22-25]. In this work, we present our long-term observations that the sedimentation influence on the crystallization process of charged colloidal particles using the density matching method.

\section{EXPERIMENTS}

\section{A. Materials}

The negatively charged polystyrene (PS) particles used in this work were synthesized by an emulsion polymerization method [41]. Thereafter, purification of the spheres was performed by filtration and followed by repeated washing with fresh distilled water in the centrifugation process. The latex was stored with resin (AG501-X8(D), Bio-Rad Laboratories, USA) for further use. The mean diameter and polydispersity of the particles determined by dynamic light scattering are $98 \mathrm{~nm}$ and 5\%, respectively. The analytical charge density is $6.9 \mu \mathrm{C} / \mathrm{cm}^{2}$ according to conductometric titration. The suspensions used in this study were prepared by carefully mixing the as-prepared latex with a certain amount of pure water or heavy water, and then the mixtures were sonicated for several minutes to attain homogeneous ones. The densities of $\mathrm{H}_{2} \mathrm{O}$, PS particles, and $\mathrm{D}_{2} \mathrm{O}$ are approximately 1.00, 1.05, and $1.10 \mathrm{~g} / \mathrm{cm}^{3}$ respectively, at room temperature $(25$ $\left.{ }^{\circ} \mathrm{C}\right)$. The density of a $50 \% \mathrm{H}_{2} \mathrm{O}$ plus $50 \% \mathrm{D}_{2} \mathrm{O}$ mixture as the liquid phase was just matched to the density of

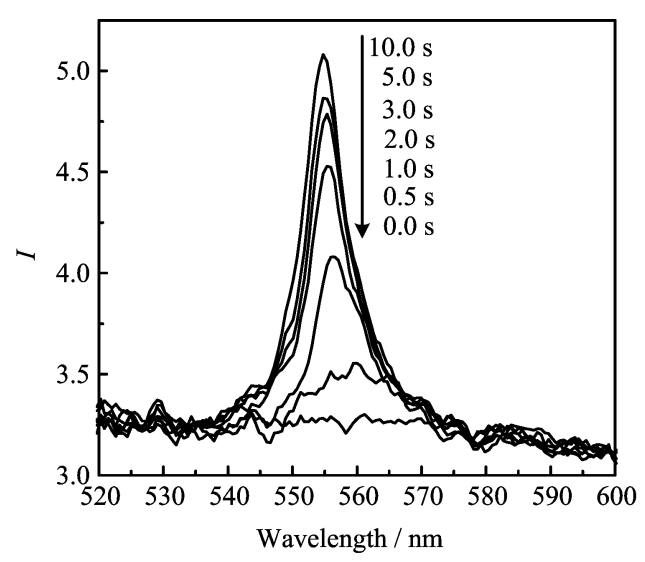

FIG. 1 Reflection spectra in the course of crystallization of sample at $25{ }^{\circ} \mathrm{C}, \Phi=0.6$.

the PS particles. Therefore when the PS particles are dispersed in $50 \% \mathrm{H}_{2} \mathrm{O}$ plus $50 \% \mathrm{D}_{2} \mathrm{O}$ mixture, sedimentation should be stopped. In this work, PS suspensions in the solvent mixture with different particle volume fractions ( $\Phi=0.4$ and 0.6 ) were placed in crystallization cell, then the density-matched (almost no sedimentation takes place or equivalent to the microgravity condition) and density-unmatched (equivalent to the normal gravity condition or under the normal gravity's acceleration) experiments of charged colloidal particles are compared to examine the influence of sedimentation on the crystallization process.

\section{B. Reflection spectroscopy}

The experimental setup used in this work is the same as that described in our previous works [42-44]. This system included a suspension circulation system that consisted mainly of a crystallization cell, a reflection spectrometer, a circulating pump, an ion-exchange chamber, and a conductivity measurement unit. A fiberoptic spectrometer (Avaspec-2048, Avantes, Netherlands) with a tungsten halogen light source (Avalight-HAL, Avantes, Netherlands) and a bifurcated fiber optic cable were used to scan the light intensity reflected from the crystallization cell over a certain wavelength range. Data including the distribution of reflection intensity vs. wavelength were recorded at the rate of four spectra per second. The peak reflection intensity increased and the peaks became sharper with time after the shear flow stopped. For a typical example, see Fig.1.

\section{Analysis of the reflection spectroscopy}

As discussed in Ref.[24], considering the reflection spectroscopy of the sample, the peak intensity of reflection spectra $I$, is related to the size of the colloidal single crystals $L$, and the number of single crystals in 


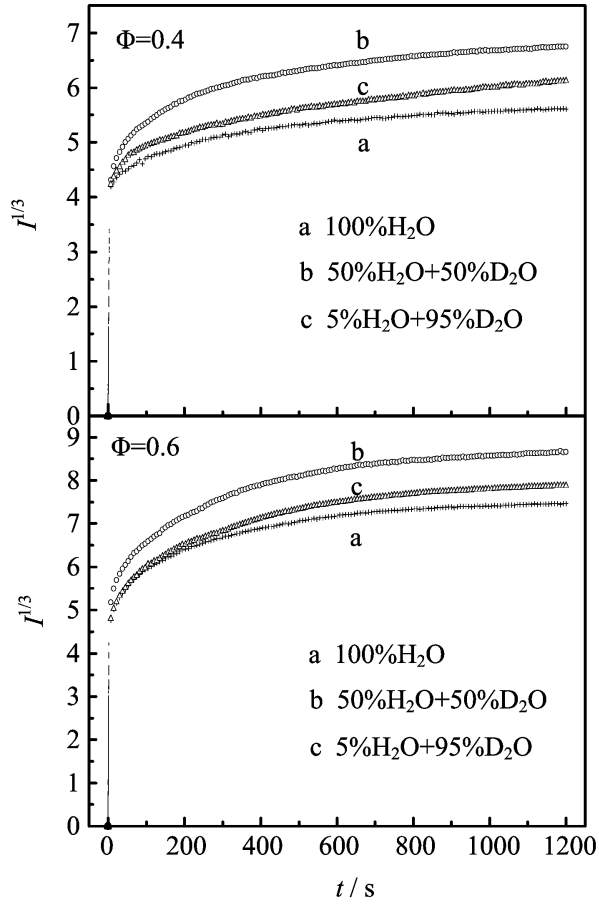

(A)

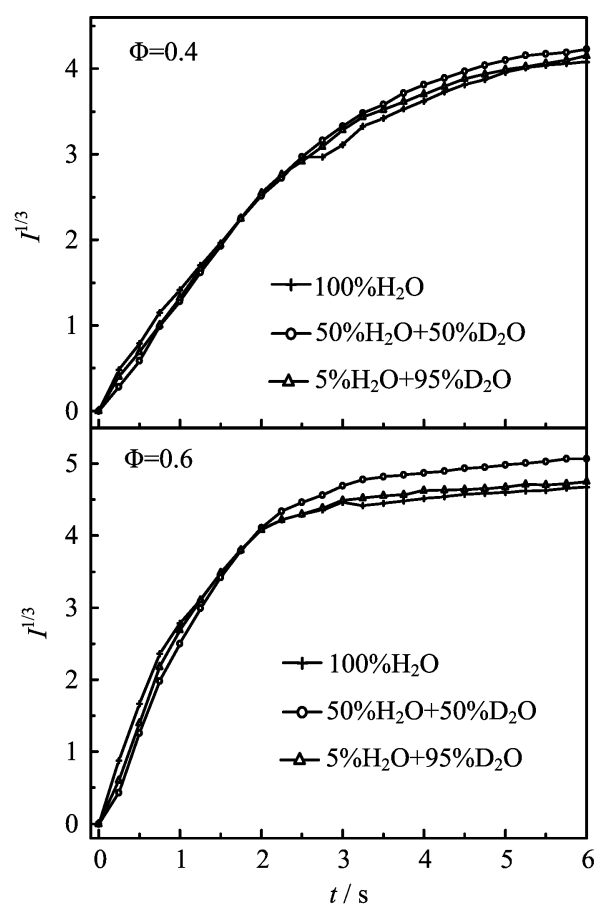

(B)

FIG. 2 (A) Cube roots of the reflection intensity in the course of crystallization of samples. (B) An enlarged view of the first $6 \mathrm{~s}$ portion of the plot in (A).

the reflecting volume $N_{\text {cryst }}$, which is directly proportional to the number concentration of crystals in the final stage of crystallization process, being equal to the total number of nuclei which were formed in the whole course of crystallization. The relationship between $I$ and $L$ can be expressed by the following equation:

$$
\begin{aligned}
& I \propto N_{\text {cryst }} L^{3} \propto L^{3} \\
& L \propto I^{1 / 3}
\end{aligned}
$$

Therefore, the crystallization evolution can be estimated from a series of peak reflection intensities of the tested sample in time sequence.

In this study, each experimental observation of the colloidal crystallization lasted $1200 \mathrm{~s}$. To reduce random uncertainty on results of experimental measurements, for each mixing formula of solvent media, five different samples were tested and for each sample ten independent observational runs of recrystallization process were performed to yield an average result. The associated experimental standard deviation is estimated to be $5 \%$.

\section{RESULTS AND DISCUSSION}

The relatively stable microgravity environment created by the density-matching method provides a favorable condition for studying the effect of the sedimentation on the colloidal crystallization process.
In the following we will use $g \approx 0$ (denoting under the microgravity condition) and $g \approx 1$ (under normal gravity condition), to represent the density-matched and unmatched cases, respectively.

The plot of the cube roots of peak reflection intensity (namely, the relative size, $L$, of the crystals according to Eq. (2)) for the density-matched $(g \approx 0)$ and -unmatched $(g \approx 1)$ suspensions $v s$. time $t$ during the crystallization is given in Fig.2. We took $t=0$ at the moment when the peristaltic pump was stopped. The rate of crystal growth is the rate at which the linear dimension of crystallites increases [45]. Therefore, this rate can also be estimated from the slope of the curve of the cube root of the peak intensity vs. time.

In Fig.2, we used the cube roots of peak-reflection intensity for the density-matched medium. Figure 2 shows that the colloidal crystal growth has two distinct stages: an initial fast growth followed by a slow growth. Figure 2(B) is an enlarged view of the first 6 -s portion of the plot in Fig.2(A). Since the particle source is limited, we can see the pattern of fast crystal growth initially, followed by slow growth, and a final stage where crystal size approaches a constant value. The changing tendency of reflection peak intensities over time is similar to that of colloidal crystallization of silica spheres [24].

Comparing the data points for $g \approx 0$ and $g \approx 1$ cases, it is clearly shown that at the beginning, the crystal size for $g \approx 1$ grows faster than that for $g \approx 0$. Then, crystal size growth for the $g \approx 0$ case became faster and finally overtook and outstripped that of the $g \approx 1$ case. 
Our observation that at the beginning of the crystallization, the crystal growth rate for the $g \approx 1$ case is larger than that for the $g \approx 0$ case, which coincides with the finding of colloidal crystallization with silica spheres in parabolic flight experiments [24]. Meanwhile, during the middle stage of crystallization, the higher crystal growth rate for the $g \approx 0$ case was also observed in the experiments with latex particles using sounding rocket [23]. The difference is that with the same particles in the same experiment, we found a two-fold effect of gravity: acceleration and retarding of crystal growth.

Our explanation for this phenomenon is that the sedimentation plays different roles in different stages of the crystal growth process. The settling velocity is an important factor. From the balance between gravitational force, buoyancy, and viscous drag, we can obtain the settling velocity $v$ of an assumed spherical crystal with a radius $R$, which can be approximated by

$$
v=\frac{2 \Delta \rho g R^{2}}{9 \eta}
$$

where $\Delta \rho$ is the difference in density between crystal and liquid phases, $g$ is gravity's acceleration with the actual value of about $9.8 \mathrm{~m} / \mathrm{s}^{2}$, and $\eta$ is the viscosity of water. So the settling velocity increases in proportion to the squared value of the crystal radius.

At early stage of the crystallization sequence, as crystals are growing, large crystals settle faster and may capture more particles underneath during sedimentation than that by diffusion alone (without sedimentation). When crystal sizes increase further, the settling velocities are also increasing and hydrodynamic friction may cause some particles to fall off from the crystal structures because particles in the colloidal crystal phase are only weakly bonded. Therefore, roughly speaking, with reference to the physical picture associated with the observation, the role of sedimentation varies in the crystallization course for the $g \approx 1$ case: as the crystal grows in size, sedimentation starts to accelerate the crystal growth for a short period and then retards further growth of the crystal. On the other hand, for the $g \approx 0$ case, crystal growth relies only on particles' diffusion and the crystal sizes grow at a steadier pace.

The differences in the crystal growth rates obtained from the experiments with density-matched and density-unmatched media is confirmed only by the sedimentation, not any other factor, the density-unmatched experiments of the suspension with solvent mixture of $5 \% \mathrm{H}_{2} \mathrm{O}+95 \% \mathrm{D}_{2} \mathrm{O}$ were also performed. According to Eq.(3) and the densities of $\mathrm{H}_{2} \mathrm{O}$, PS particles and $\mathrm{D}_{2} \mathrm{O}$, the settling speed of a particle in $100 \% \mathrm{H}_{2} \mathrm{O}$ should be equal to its floating up speed in $100 \% \mathrm{D}_{2} \mathrm{O}$. Therefore, compared with the densitymatched (in $50 \% \mathrm{H}_{2} \mathrm{O}+50 \% \mathrm{D}_{2} \mathrm{O}$ ) experiments of crystal size vs. time, the two density-unmatched (namely, in $100 \% \mathrm{H}_{2} \mathrm{O}$ and $100 \% \mathrm{D}_{2} \mathrm{O}$, respectively) experiments should basically show similar behavior. Since the PS particles were originally dispersed in $\mathrm{H}_{2} \mathrm{O}$, we lacked an efficient way to use $\mathrm{D}_{2} \mathrm{O}$ to replace all $\mathrm{H}_{2} \mathrm{O}$ in the final dispersion. Therefore we used the suspension with solvent mixture of $5 \% \mathrm{H}_{2} \mathrm{O}+95 \% \mathrm{D}_{2} \mathrm{O}$, instead of $100 \% \mathrm{D}_{2} \mathrm{O}$ solvent. We suppose that this $5 \%$ shortage of $\mathrm{D}_{2} \mathrm{O}$ should not yield significant difference in the experimental result.

From Fig.2, we can see that $100 \% \mathrm{H}_{2} \mathrm{O}$ and $95 \% \mathrm{D}_{2} \mathrm{O}$ do basically share similar behavior in comparison with the density-matched case, $50 \% \mathrm{H}_{2} \mathrm{O}+50 \% \mathrm{D}_{2} \mathrm{O}$. However, the difference between $95 \% \mathrm{D}_{2} \mathrm{O}$ and $50 \% \mathrm{H}_{2} \mathrm{O}+50 \% \mathrm{D}_{2} \mathrm{O}$ seems to be too distinguishable. Apparently, this difference can not be simply interpreted as the cause of using a solvent mixture of $5 \% \mathrm{H}_{2} \mathrm{O}+95 \% \mathrm{D}_{2} \mathrm{O}$ as a substitute for a $100 \% \mathrm{D}_{2} \mathrm{O}$ solvent.

In Fig.2(B), it can be seen that at $t=0.25 \mathrm{~s}$ the crystal size grown in $95 \% \mathrm{D}_{2} \mathrm{O}$ solvent is obviously smaller than that in $100 \% \mathrm{H}_{2} \mathrm{O}$ solvent. We suppose that it is because the viscosities $(\eta)$ of water and heavy water are different. The viscosity of a mixture composed of $5 \% \mathrm{H}_{2} \mathrm{O}+95 \% \mathrm{D}_{2} \mathrm{O}$ is about 1.21 times larger than that of $100 \% \mathrm{H}_{2} \mathrm{O}$ at $25{ }^{\circ} \mathrm{C}$. In this case, according to Eq.(3), the downward settling speed of a large crystal in water is greater than its upward floating speed in heavy water. In this case, when large crystals settle in water, they may capture particles underneath them. The numbers of particles they capture should be more than that they capture above them when they are floating up in heavy water. Therefore, we can see that at the early stage of the crystallization sequence, the size of crystals grown in water is larger than that in heavy water, as shown at $t=0.25 \mathrm{~s}$ in Fig. $2(\mathrm{~B})$.

On the other hand, as discussed earlier, at the late stage of the crystallization, with the crystal size growing, faster moving crystals (larger) may lose more particles than slower ones do. Therefore, the size of crystals grown in water is smaller than that in heavy water because crystals move faster and thus lose more particles in water. This should explain why $5 \% \mathrm{H}_{2} \mathrm{O}+95 \% \mathrm{D}_{2} \mathrm{O}$ is above $100 \% \mathrm{H}_{2} \mathrm{O}$ when $t>2.5 \mathrm{~s}$ for $\Phi=0.4$ and $t>3 \mathrm{~s}$ for $\Phi=0.6$, as shown in Fig. 2 .

As an alternative to the method of the cube roots of peak-reflection intensity of Eq.(2), the Scherrer equation [36] is also quite often to be used to estimate the average crystallite size. In this case, the average size of crystals $L$ is calculated by the relationship:

$$
\begin{aligned}
L & =\frac{2 \pi k}{\Delta q(t)} \\
& =m \frac{k \lambda_{\mathrm{L}} \lambda_{\mathrm{S}}}{2 n\left(\lambda_{\mathrm{L}}-\lambda_{\mathrm{S}}\right)}
\end{aligned}
$$

where $\Delta q(t)$ is full width at half-maximum of the peak, $k=1.155$ is the Scherrer constant for a crystal of cubic shape. In addition, $m, \lambda$ and $n$ represent the degree of Bragg's reflection, the spectral peak wavelength, the refractive index, respectively. $\lambda_{\mathrm{L}}$ and $\lambda_{\mathrm{S}}$ mean the larger and the smaller wavelength at the half-width of the reflection peaks in Fig.1. The crystal sizes calculated from 


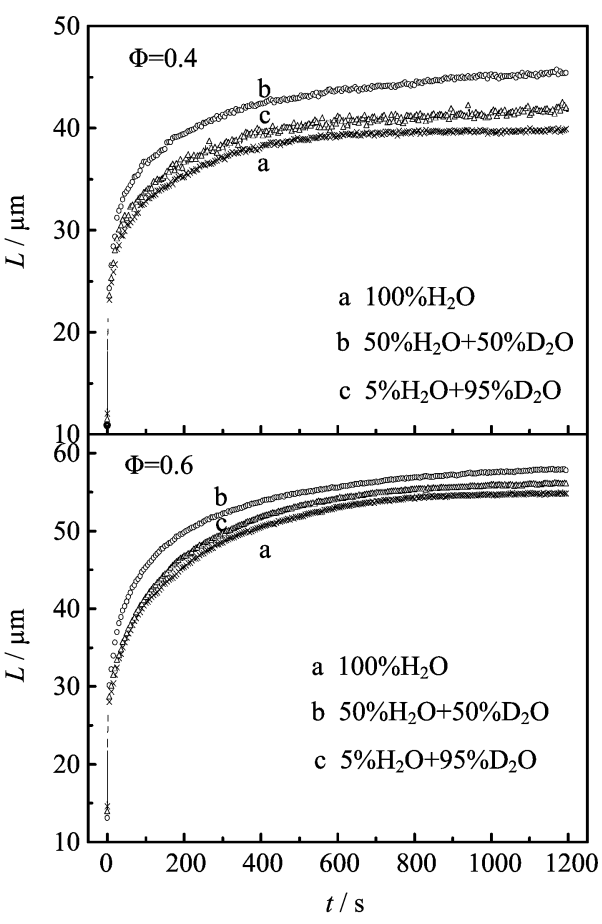

(A)

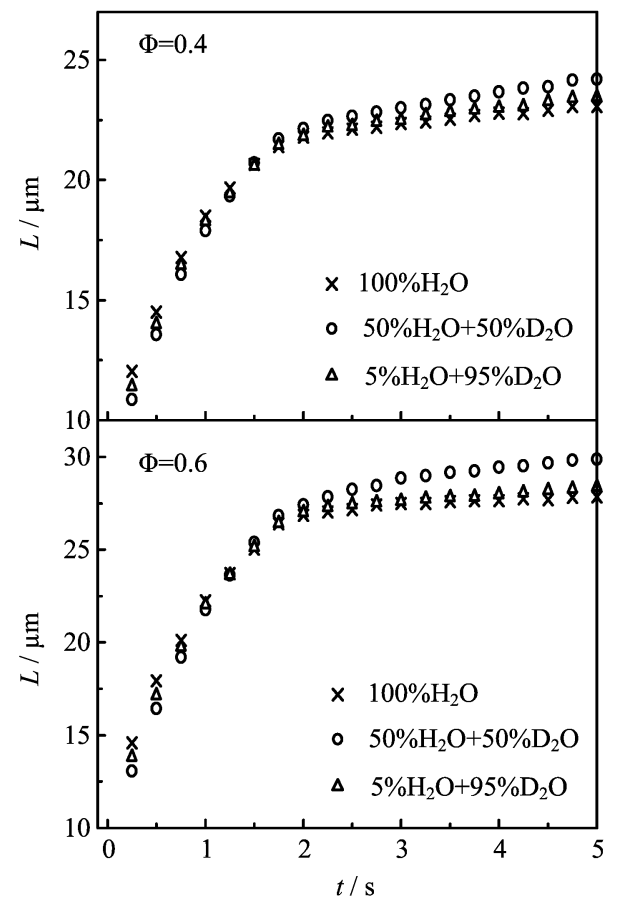

(B)

FIG. 3 (A) Time development of the average crystallite dimensions for samples. The size of crystals were evaluated using the Sherrer's method. (B) An enlarged view of the first $5 \mathrm{~s}$ portion of the plot in (A).

the half-width method using Eqs. (1) and (2) are shown in Fig.3 as a function of time. The range of growing crystals size is from $10 \mu \mathrm{m}$ to $45 \mu \mathrm{m}$ for sample $\Phi=0.4$, and from $12 \mu \mathrm{m}$ to $57 \mu \mathrm{m}$ for sample $\Phi=0.6$. As can be seen from Fig. 3, all the samples show fast and significant increase in the early stage. The crystal size increased linearly at the beginning of the crystal growth process. Then, the average crystal size grows more slowly during the main crystallization process, and the later ripening stage. In addition, it is shown in Fig. $3(\mathrm{~A})$ and $(\mathrm{B})$ that $L$ for $g \approx 0$ grows slower than that for $g \approx 1$ at the beginning. But it grows faster for the $g \approx 0$ case and surpasses that of the $g \approx 1$ case within $1 \mathrm{~s}$. This result is consistent with that shown in Fig.2 that is calculated Eq.(2).

In this study, we did not attempt to evaluate the nucleation rates by measuring the induction period of the crystallization as described in Ref.[24] because a higher suspension concentration was used due to the limited degree of deionization of the solution. A short induction period makes accurate measurement difficult. However, it can be seen from Fig. 2 that crystal growth behavior presented in this work is consistent with that of Ref.[24] after induction period.

The interparticle distance $D_{0}$ of colloidal crystals can be obtained from Bragg's diffraction condition by measuring the reflection spectrum:

$$
m^{2} \lambda^{2}=\frac{8}{3} D_{0}^{2}\left(n_{\mathrm{s}}{ }^{2}-n_{\mathrm{a}}{ }^{2} \cos ^{2} \theta\right)
$$

where $m, \lambda, n_{\mathrm{s}}, n_{\mathrm{a}}$ and $\theta$ represent the degree of
Bragg's reflection, the spectral peak wavelength, the refractive index of the sample, the refractive index of air and the Bragg reflection angle, respectively. In our case, $m=2, \theta=90^{\circ}, n_{\mathrm{s}}\left(100 \% \mathrm{H}_{2} \mathrm{O}\right)=1.3331$ and $n_{\mathrm{s}}\left(50 \% \mathrm{H}_{2} \mathrm{O}+50 \% \mathrm{D}_{2} \mathrm{O}\right)=1.3305$. An example of the time dependencies of the interparticle distance $D_{0}$ is shown in Fig.4. After stopping the shear flow in the sample cell, value of $D_{0}$ dropped drastically first and then gently approached a constant value with time. This behavior supports the assumption that metastable and expanded structures are formed first in the crystallization process and then the crystal structures become more stable and more closely packed. Figure 4 also shows that the interparticle distances of the density-matched and -unmatched samples were quite similar at the very initial stage of crystallization. However, the interparticle distance for the $g \approx 0$ case, was smaller than that for the $g \approx 1$ case in the later stage of crystal growth process. This experimental observation suggests that crystal structures formed under microgravity were more closely packed than that in gravity.

\section{CONCLUSION}

In this work, we made several efforts aimed at examining the influence of sedimentation due to gravity on the crystallization of charged colloidal particles. First, the density-matching method was adopted to eliminate 

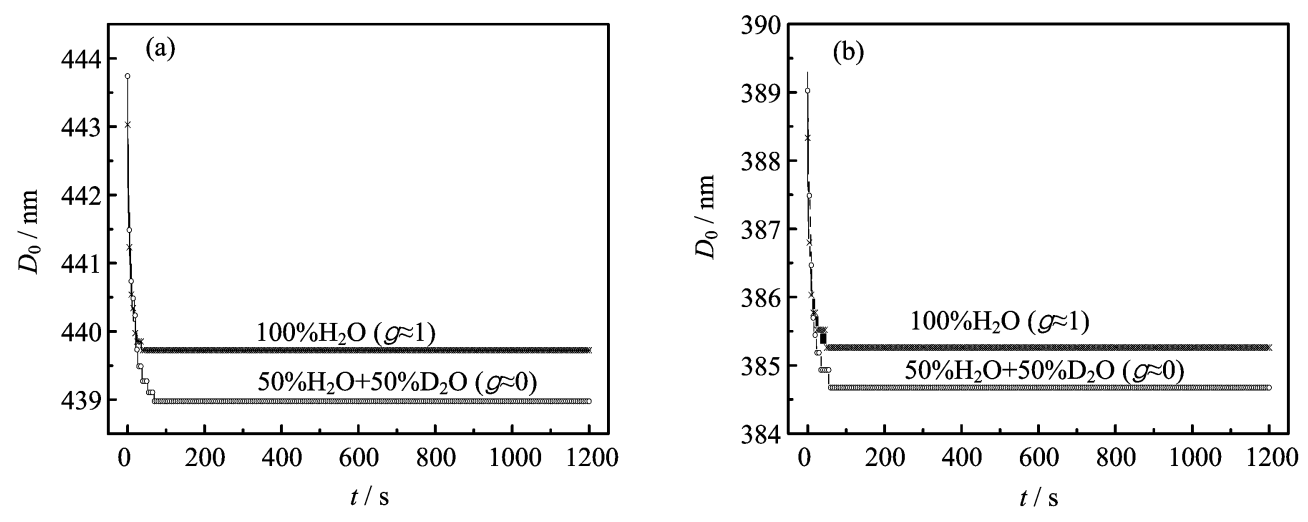

FIG. 4 Change in the interparticle distances over the course of the crystallization of samples. (a) $\Phi=0.4$ and (b) $\Phi=0.6$.

sedimentation and reduce the temperature gradient on experimental samples as much as possible. Through this approach, we achieved an equivalent, long-lasting microgravity condition.

We used the reflection spectra of colloidal suspensions to monitor crystal growth. Comparing the data obtained from the density-matched experiments $(g \approx 0)$ with those from the density-unmatched ones $(g \approx 1)$, the influence of sedimentation on crystallization were analyzed and discussed. Our experiments showed that: (i) sedimentation appeared to accelerate the crystal size growth initially and then retard the growth; (ii) crystal structures formed under microgravity were more closely packed than that in normal gravity.

We also offered a preliminary explanation for the above findings. It is sedimentation that causes the additional collisions of particles and aggregates, resulting in the increase of crystal growth rate. When crystals grow further, however, their settling velocities become large and hydrodynamic friction strips off particles from their delicate crystal structures.

To confirm what we found in the density-matched and -unmatched experiments is able to represent the behavior of colloidal crystal growth under $g \approx 0$ and $g \approx 1$ condition, experiment with PS particles dispersed in heavy water was performed. A similar phenomenon was also observed for the latter case.

Finally, our experimental observation suggests that crystal structures formed under microgravity were more closely packed than that in gravity. It implies that the stable environment of $g \approx 0$ (no settling motion) is more favorable for crystals to form more compact structures.

\section{ACKNOWLEDGMENTS}

This work was supported by the National Natural Science Foundation of China (No.11172302, No.10972217 and No.11032011), the Knowledge Innovation Program of the Chinese Academy of Sciences (No.KJCX2-YWL08) and the National Science Foundation for Postdoctoral Scientists of China (No.20100480482).
[1] B. J. Ackerson and N. A. Clark, Phys. Rev. Lett. 46 123 (1981).

[2] T. Okubo, J. Chem. Soc. Faraday Trans. 84, 1163 (1988).

[3] D. J. W. Aastuen, N. A. Clark, J. C. Swindal, and C. D. Muzny, Phase Transit. 21, 139 (1990).

[4] T. Yoshiyama, Polymer 27, 827 (1986).

[5] N. A. Clark, A. Hurd, and B. J. Ackerson, Nature 281, 57 (1979).

[6] T. Okubo, J. Chem. Phys. 93, 8276 (1990).

[7] T. Yoshiyama, I. Sogami, and N. Ise, Phys. Rev. Lett. 53, 2153 (1984).

[8] P. Wette, H. J. Schope, and T. Palberg, J. Chem. Phys. 123, 174902 (2005).

[9] P. Wette, H. J. Schope, and T. Palberg, J. Chem. Phys. 122, 144901 (2005).

[10] A. Tsuchida, E. Takyo, K. Taguchi, and T. Okubo, Colloid Polym. Sci. 282, 1105 (2004).

[11] T. Shinohara, T. Yoshiyama, I. S. Sogami, T. Konishi, and N. Ise, Langmuir 17, 8010 (2001).

[12] S. Hachisu and S. Yoshimura, Nature 283, 188 (1980).

[13] K. Yoshinaga, K. Fujiwara, E. Mouri, M. Ishii, and H. Nakamura, Langmuir 21, 4471 (2005).

[14] T. Shinohara, T. Yoshiyama, T. Yoshiyama, T. Itoh, I. Sogami, and N. Ise, Phys. Rev. E 70, 062401 (2004).

[15] T. Okubo and H. Ishiki, J. Colloid Interface Sci. 228, $151(2000)$.

[16] R. S. Crandall and R. Williams, Science 198, 293 (1977).

[17] K. Furusawa and N. Tomotsu, J. Colloid Interface Sci. 93, 504 (1983).

[18] J. Zhu, M. Li, R. Rogers, W. Meyer, and R. H. Ottewill, Nature 387, 883 (1997).

[19] D. A. Weitz, Science 303, 968 (2004).

[20] A. R. Bausch, M. J. Bowick, A. Cacciuto, A. D. Dinsmore, M. F. Hsu, D. R. Nelson, M. G. Nikolaides, A Travesset, and D. A. Weitz, Science 299, 1716 (2003).

[21] M. G. Nikolaides, A. R. Bausch, M. F. Hsu, A. D. Dinsmore, M. P. Brenner, C. Gay, and D. A. Weitz, Nature 420, 299 (2002).

[22] M. Ishikawa and T. Okubo, J. Cryst. Growth 233, 408 (2001).

[23] M. Ishikawa, H. Morimoto, T. Okubo, and T. Maekawa, 
Int. J. Mod. Phys. B 2, 338 (2002).

[24] T. Okubo, A. Tsuchida, T. Okuda, K. Fujitsuna, M. Ishikawa, T. Morita, and T. Tada, Colloids Surf. A. 153, 515 (1999).

[25] T. Okubo, A. Tsuchida, S. Takahashi, K. Taguchi, and M. Ishikawa, Colloid Polym. Sci. 278, 202 (2000).

[26] J. Liu, Z. W. Sun, and A. A. Yan, Chin. Phys. Lett. 22, 3199 (2005).

[27] T. Okubo, J. Chem. Phys. 102, 7721 (1995).

[28] T. Okubo, J. Phys. Chem. 98, 1472 (1994).

[29] T. Okubo, J. Chem. Phys. 86, 2394 (1987).

[30] T. Okubo, J. Chem. Phys. 87, 5528 (1987).

[31] S. E. Paulin and B. Ackerson, Phys. Rev. Lett. 64, 2663 (1990).

[32] Z. Cheng, P. M. Chaikin, J. Zhu, W. B. Russel, and W. V. Meyer, Phys. Rev. Lett. 88, 015501 (2002).

[33] J. L. Harland, S. I. Henderson, S. M. Underwood, and W. V. Megen, Phys. Rev. Lett. 75, 3572 (1995).

[34] Z. Cheng, J. Zhu, W. B. Russel, W. V. Meyer, and P. M. Chaikin, Appl. Opt. 40, 4146 (2001).

[35] H. J. Schope, G. Bryant, and W. V. Megen, Phys. Rev. Lett. 96, 175701 (2006).
[36] S. Iacopini, T. Palberg, and H. J. Schope, J. Chem. Phys. 130, 084502 (2009).

[37] H. Yoshida, K. Ito, and N. Ise, J. Chem. Soc. Faraday Trans. 87, 371 (1991).

[38] A. Yethiraj and A. van Blaaderen, Nature 421, 513 (2003).

[39] W. K. Kegel and A. van Blaaderen, Science 287, 290 (2000).

[40] J. Liu, Z. W. Sun, and A. A. Yan, Chin. Phys. Lett. 22, 3199 (2005).

[41] G. T. D. Shouldice, G. A. Vandezande, and A. Rudin, Eur. Polym. J. 30, 179 (1994).

[42] H. W. Zhou, S. H. Xu, Z. W. Sun, X. Du, and J. C. Xie, Colloids Surf. A 375, 50 (2011)

[43] S. H. Xu, H. W. Zhou, Z. W. Sun, and J. C. Xie, Phys. Rev. E 82, 010401 (2010).

[44] H. W. Zhou, S. H. Xu, Z. W. Sun, X. Du, and L. X. Liu, Langmuir 27, 7439 (2011).

[45] J. K. J. Dhont, C. Smits, and H. N. W. Lekkerkerker, J. Colloid Interface Sci. 152, 386 (1992). 